\title{
Arterial spasm and slowing of the cerebral circulation in the ischaemia of head injury
}

\author{
P. MACPHERSON AND D. I. GRAHAM
}

\begin{abstract}
From the Division of Neuroradiology and the University Department of Neuropathology,
\end{abstract} The Institute of Neurological Sciences, Glasgow

\begin{abstract}
SUMMARY Carotid angiograms of 33 patients who had died during 1968 and 1969 from blunt head injury were reviewed and assessed for evidence of arterial spasm and slowing of the cerebral circulation. Spasm was found in $57.5 \%$, a prolonged circulation time in $57.5 \%$, and a combination of both features in $42.4 \%$ of cases. In the same group of patients there was also a high incidence of ischaemic brain damage. There appeared to be some correlation between arterial spasm and ischaemic damage in the cerebral cortex, but none in the basal ganglia or in the white matter. There was no apparent correlation between a prolonged cerebral circulation time and ischaemic brain damage.
\end{abstract}

Recent studies in this Institute have established that there is a high incidence of ischaemic brain damage in patients dying after blunt head injury (Graham and Adams, 1971; Jennett et al., 1972). It has been suggested that a reduced cerebral blood flow is the major factor leading to this type of brain damage, and that such a reduction is more likely to result from an increase in intracranial pressure than from a fall in the systemic arterial pressure (Jennett et al., 1972). However, in view of the known occurrence of spasm in cerebral arteries in head-injured patients (Wilkins and Odom, 1970; Suwanwela and Suwanwela, 1972) and of slowing of the cerebral circulation with raised intracranial pressure (Davies and Sutton, 1967), we decided to investigate the association between these two findings of arterial spasm and slowing of the circulation as shown by carotid angiography, and the occurrence of ischaemic brain damage in a group of headinjured patients.

\section{METHODS}

The series comprised all 33 patients who died in this Institute after head injury during 1968 and 1969, on whom carotid angiography had been carried out before any surgical treatment, and on whom a full neuropathological examination was subsequently performed. There were 26 males and seven females, aged from 5 to 73 years; the survival after injury ranged from six hours to seven months.

ANGIOGRAPHY This examination was carried out under general anaesthesia using controlled ventilation. Contrast medium $(10 \mathrm{ml}$.) was injected by hand through a 17 gauge cannula into the common carotid artery. Serial films were taken on the Barr and Stroud manual cassette changer using an automatic timing device giving $1.25 \mathrm{sec}$ between each exposure.

ARTERIAL SPASM Arterial narrowing was attributed to spasm when there was a localized or diffuse smooth and circumferential decrease in the calibre of the vessels, or where segmental narrowing gave a beaded appearance on the angiogram (Wilkins and Odom, 1970). Any appearance which suggested that the narrowing might be due to an anatomical variant, atheroma, or stretching of vessels was not included in the category of spasm.

The spasm was divided into three categories: (1) basal, when it involved the upper end of the internal carotid artery and the proximal parts of the anterior and middle cerebral arteries; (2) peripheral, when it involved the distal parts of the anterior and middle cerebral arteries; and (3) generalized when there was a diffuse and uniform reduction in the size of all the vessels.

CIRCULATION TIME In a series of 50 consecutive intracarotid injections, the average injection time was just under $1 \mathrm{sec}$ (longest $1.6 \mathrm{sec}$ ). The first film was 
taken on average $0.2 \mathrm{sec}$ after the end of the injection. If the contrast medium was still present in the arteries in the third film ( $>2.5 \mathrm{sec}$ after injection) the 'general arterial circulation time' (Leeds and Taveras, 1963) was recorded as slightly prolonged, if in the fourth film $(>3.75 \mathrm{sec})$ moderately prolonged, and in the fifth film $(>5.0 \mathrm{sec})$ markedly prolonged.

NEUROPATHOLOGY All the brains were fixed for at least three weeks in $10 \%$ formol saline before dissection. Large bilateral blocks were taken from the cerebral and cerebellar hemispheres and from the brain-stem. They were embedded in celloidin and sections cut at $30 \mu \mathrm{m}$, stained by the method of Nissl using cresyl violet and by Woelke's modification of Heidenhain's method for myelin, and examined microscopically.

\section{RESULTS}

There was arterial spasm in 19 of the 33 cases $(57.5 \%)$, slowing of the circulation in $19(57.5 \%)$, and spasm combined with slowing in $14(42 \cdot 4 \%)$. In only nine cases were the angiograms normal.

There was ischaemic damage in the basal ganglia in 30 cases and in the white matter of the cerebral hemispheres in 31. Excluding infarction related to contusions, cardiorespiratory arrest, fat embolism and that within the distribution of the posterior cerebral artery, there was ischaemic damage in the cortex in 14 of the 33 cases; in two there were multiple foci of necrosis, in six there was infarction in specific arterial territories, and in six infarction in the arterial boundary zones. The cases with ischaemic damage in the cortex form a particularly interesting group as it seems unlikely that this type of damage can be attributed to brain shift, distortion, and raised intracranial pressure (Jennett et al., 1972). We have therefore paid particular attention to the association between ischaemic damage in the cortex and angiographic evidence of arterial spasm and slow circulation. There was only one case without ischaemic damage in the brain.

ARTERIAL SPASM (19 cases) There was ischaemic damage in the cortex in nine of the cases with arterial spasm (Table). In one of these cases basal and generalized spasm was associated with multiple foci of ischaemic necrosis; in two cases with spasm of basal and peripheral vessels there was infarction within the appropriate arterial territories of supply; in one case with
TABLE

ASSOCIATION BETWEEN SPASM, SLOW CEREBRAL CIRCULATION, AND ISCHAEMIC BRAIN DAMAGE

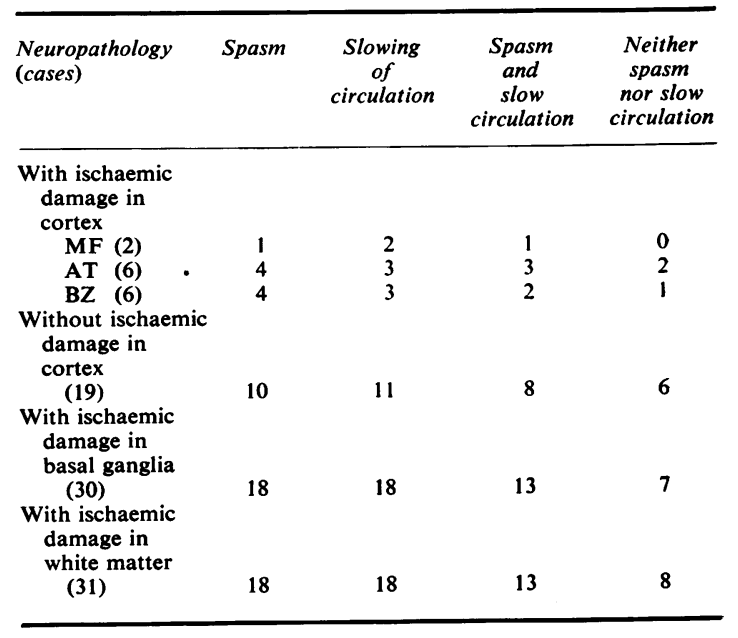

spasm of a peripheral vessel there was infarction within its territory of supply; in one case witlp spasm of basal vessels there was an arteriab territory infarct; and in four cases-two witlo basal and two with basal and peripheral spasm? there was infarction within the arterial boundar zones. In 10 cases there was spasm of various vessels but no evidence of ischaemic damage in the cortex. There was no evidence of arterial spasm in the remaining five cases with cortical brain damage.

In the 18 cases in which arterial spasm was associated with ischaemic damage in the basal ganglia and white matter, there was no correlation between the localization of the spasm and the extent and distribution of the ischaemic changes.

SLOWING OF THE CIRCULATION (19 cases) The degree was slight in 10, moderate in two, and marked in seven. In eight of the 19 cases there was ischaemic brain damage in the cortex (Table). In two cases slowing was associated with multiple foci of ischaemic necrosis, in three cases there was infarction within arterial territories, and in three cases there was infarction within arterial boundary zones. In 11 cases there were various grades of slowing but no evidence of cortical brain damage. There were 
six cases with ischaemic damage in the cortex in which no prolongation of the circulation time was identified. In the 18 cases in which slowing of the crrculation was associated with ischaemic damage in the basal ganglia and white matter, there was no apparent correlation between the degree of slowing and the extent and distribution of the ischaemic changes.

ARTERIAL SPASM AND SLOWING OF THE CIRCULATION (14 cases) There was ischaemic damage in the cortex of six cases. In one with arterial spasm (basal and generalized) and a marked slowing of the circulation, there were multiple foci of necrosis. In one with spasm (basal and peripheral middle cerebral) and marked slowing, there was an infarct within the distribution of the middle cerebral artery. In four cases there was no apparent association between spasm together with prolonged circulation time and the type and distribution of the cortical brain damage. There were therefore eight cases with arterial spasm and a slow circulation in which there was no ischaemic damage in the cortex. There were three cases with ischaemic damage in the cortex in which neither spasm nor slowing of the circulation was present. In the 13 cases with arterial spasm and a slow circulation in which there was ischaemic damage either in the basal ganglia or the white matter, there was no apparent correlation between the angiographic appearances and the extent and distribution of the ischaemic damage.

\section{DISCUSSION}

Narrowing of the cerebral vessels in cases with head injury is becoming increasingly recognized (Schechter and Zingesser, 1966; Wilkins and Odom, 1970) and Suwanwela and Suwanwela (1972) have recently surveyed the literature and found the incidence to range from $2-31 \%$. In the present series narrowing of vessels attributable to spasm was present in $57.5 \%$ of cases. Whereas patients with arterial spasm due to spontaneous subarachnoid haemorrhage may have a reduction in cerebral blood flow and an abnormal brain scan (Wilkins et al., 1972) and at necropsy have evidence of ischaemic brain damage (Tomlinson, 1959), similar information in headinjured patients is lacking. However, Wilkins and Odom (1970) have shown that arterial spasm after head injury may be associated with a neurological deficit, and Suwanwela and Suwanwela (1972) believe that such spasm can influence the clinical course and prognosis.

The incidence of arterial spasm in the present series is very high $(57.5 \%)$, and in six of the 14 cases with cortical brain damage there was a correlation between the ischaemic damage and spasm of the appropriate cerebral arteries. However, there was no apparent correlation between arterial spasm and ischaemic damage in the basal ganglia and the white matter. The high incidence of arterial spasm in this fatal group of headinjured patients suggests that, at least in some cases, spasm may influence the prognosis.

Delayed filling of cerebral vessels has been noted in head-injured patients (Leeds et al., 1966) and reproduced in experimental head trauma (Rockoff and Ommaya, 1964). A slow cerebral circulation may be due to a number of factors (Schechter and Zingesser, 1966), but in most instances can be taken as evidence of raised intracranial pressure (Davies and Sutton, 1967; Swischuk et al., 1972). Angiographic evidence of prolonged circulation time - and hence presumably of raised intracranial pressure-was present in $57.5 \%$ of cases, but there was conventional neuropathological evidence of raised intracranial pressure in 27 of the 33 cases $(81 \cdot 8 \%)$. Such apparent discrepancies can probably be accounted for by the marked variation in injury-to-angiography and angiographyto-death times, as the angiogram simply reflects one moment in time in what is essentially a dynamic sequence of events. It is, however, surprising that in these cases in which there was a prolonged circulation time, no correlation was found between the slow circulation and the extent and distribution of the ischaemic damage.

With the possible exception of a single case with multiple foci of ischaemic damage, and one with an infarct in an arterial territory, there seemed to be little correlation between the presence of arterial spasm together with a prolonged circulation time and the amount and distribution of any ischaemic brain damage.

The results of this rather small series of cases are preliminary and somewhat inconclusive, but we believe that there is sufficient evidence to suggest that at least in some cases arterial spasm may be correlated with the type and distribution 
of ischaemic damage in the cortex. Although the ischaemic damage is probably multi-factorial in origin, it seems likely to us that increasing attention should be paid to the presence of arterial spasm in a patient with a head injury.

We would like to thank our colleagues in the Institute for making their records available and express indebtedness to Professor W. B. Jennett, Professor J. H. Adams, and Dr. J. L. Steven for help with the preparation of this paper.

\section{REFERENCES}

Davies, E. R., and Sutton, D. (1967). Pseudo-occlusion of the internal carotid artery in raised intracranial pressure. Clinical Radiology, 18, 245-252.

Graham, D. I., and Adams, J. H. (1971). Ischaemic brain damage in fatal head injuries. Lancet, 1, 265-266.

Jennett, B., Graham, D. I., Adams, H., and Johnston, I. H. (1973). Cerebral Vascular Diseases, Eighth Conference, pp. 163-170. In Proceedings of the Conference on CerebroVascular Disease, Princeton, U.S.A. Edited by F. H. McDowell and R. W. Brennan. Grune and Stratton: New York. 1973.

Leeds, N. E., Reid, N. D., and Rosen, L. M. (1966). Angio- graphic changes in cerebral contusions and intracerebral hematomas. Acta Radiologica. Diagnosis, 5, 320-327.

Leeds, N. E., and Taveras, J. M. (1963). Changes in local circulation time in frontal and parietal tumors studied by rapid serial angiography. Acta Radiologica. Diagnosis, 1, 332-342.

Rockoff, S. D., and Ommaya, A. K. (1964). Experimental head trauma. Cerebral angiographic observations in the early post-traumatic period. American Journal of Roentgenology, 91, 1026-1035.

Schechter, M. M., and Zingesser, L. H. (1966). Special procedures in the management of traumatic lesions of the head and neck. Radiologic Clinics of North America, 4, 53-74.

Suwanwela, C., and Suwanwela, N. (1972). Intracranial arterial narrowing and spasm in acute head injury. Journal of Neurosurgery, 36, 314-323.

Swischuk, L. E., Meyer, G. A., and Bryan, N. (1972). Infantile hydrocephalus and cerebral angiography. American Journal of Roentgenology, 115, 50-61.

Tomlinson, B. E. (1959). Brain changes in ruptured intracranial aneurysm. Journal of Clinical Pathology, 12, 391399.

Wilkins, R. H., and Odom, G. L. (1970). Intracranial arterial spasm associated with craniocerebral trauma. Journal of Neurosurgery, 32, 626-633.

Wilkins, R. H., Wilkinson. R. H., and Odom, G. L. (1972). Abnormal brain scans in patients with cerebral arterial 9 spasm. Journal of Neurosurgery, 36, 133-140. 